\title{
Pseudospark-based electron beam and Cherenkov maser experiments
}

\author{
H. Yin, G. R. M. Robb, W. He, A. D. R. Phelps, A. W. Cross, and K. Ronald \\ Department of Physics and Applied Physics, University of Strathclyde, Glasgow, G4 ONG, United Kingdom
}

(Received 13 April 2000; accepted 28 August 2000)

\begin{abstract}
Detailed experimental results from the first free-electron maser experiment to use a pseudospark-based electron beam are presented in this paper. These include the design and realization of a pseudospark-based electron beam source and Cherenkov maser experiment. A pulsed, 70-80 kV, 10 A electron beam was obtained from the hollow cathode discharge phase of an 8 -gap pseudospark (PS) discharge. The beam was used to produce coherent microwave radiation via a Cherenkov interaction between the electron beam and the $\mathrm{TM}_{01}$ mode of a $60-\mathrm{cm}$ long alumina-lined waveguide. A gain of $29 \pm 3 \mathrm{~dB}$ was measured and an output power of $2 \pm 0.2 \mathrm{~kW}$ in the frequency range 25.5-28.6 GHz. Results from numerical simulations of the Cherenkov amplification are also presented and found to be consistent with the experimental results. (C) 2000 American Institute of Physics. [S1070-664X(00)02412-5]
\end{abstract}

\section{INTRODUCTION}

Free-electron masers have been of general interest for over 20 years and discharges have been studied for much longer. The pseudospark discharge is, however, a more recently recognized class of discharge $e^{1,2}$ capable of producing electron beams with the highest combined current density and brightness of any known type of electron source.

A pseudospark discharge is a low pressure gas discharge in a hollow cathode and a planar anode configuration operating on the left-hand branch of a characteristic breakdown curve which is similar to the Paschen curve for parallel electrodes. It is distinguished from other discharge modes by two main interesting features: (1) The extremely rapid (and triggerable) current rise (rise time typically several tens or hundreds of ns) after application of a voltage; (2) the generation of electron and ion beams during both the current rise and final high current phase. The pseudospark discharge offers the possibility of fast, high repetition rate, high power closing switches ${ }^{3,4}$ and electron beam sources of high current density $\left(>10^{4} \mathrm{~A} \mathrm{~cm}^{-2}\right.$ ), very high brightness (up to $10^{12}$ $\left.\mathrm{Am}^{-2} \mathrm{rad}^{-2}\right)$, small beam diameter $(<4 \mathrm{~mm})$, very low emittance (tens of $\mathrm{mm} \mathrm{mrad}$ ), and variable duration (tens to hundreds of ns). ${ }^{5,6}$ It is therefore potentially very attractive as an electron beam source for high power generators of electromagnetic radiation, such as free-electron lasers (FELs), ${ }^{7}$ cyclotron autoresonance masers (CARMs) ${ }^{8}$ and Cherenkov masers. $^{9-13}$

While there has been a great deal of experimental investigation of the pseudospark discharge characteristics and the microwave radiation accompanying the passage of its electron beam through a low pressure gas, ${ }^{14,15}$ the experiment reported here is different in that the pseudospark-sourced electron beam has for the first time been applied in a freeelectron maser device. ${ }^{16}$ This experiment involved a Cherenkov maser amplifier driven by an electron beam of $10 \mathrm{~A}$, 70-80 keV, and diameter $3 \mathrm{~mm}$ from an 8-gap pseudospark discharge system. A resonant Cherenkov interaction between this beam and the $\mathrm{TM}_{01}$ mode of an alumina-lined waveguide in the frequency range $25.5-28.6 \mathrm{GHz}$ allowed ampli- fication of low power broadband microwave radiation emitted by the pseudospark discharge and resulted in a measured gain of $29 \pm 3 \mathrm{~dB}$. The article is organized as follows. The experimental apparatus is described in Sec. II and Sec. III presents the experimental results. In Sec. IV, the simulations are compared with the experimental results, and finally the conclusions are drawn in Sec. V.

\section{EXPERIMENTAL SETUP}

A schematic outline of the pseudospark-based Cherenkov maser amplifier is shown in Fig. 1. The main components of the experiment are the pseudospark-based electron beam source, the magnetic field for beam transport, the Cherenkov interaction region, electrical/beam diagnostics, and the microwave launching/diagnostic system.

The design of the pseudospark discharge was determined by the conditions needed for the hollow cathode effect ${ }^{17}$ and the required holdoff voltage of the discharge gap. The pseudospark discharge chamber consists of a planar anode, a planar cathode with an adjustable cylindrical hollow cavity, and several sets of Perspex insulators and intermediate electrodes of 6.5-mm thickness. The Perspex insulator disks have inner and outer diameters of 5.5 and $120 \mathrm{~mm}$, respectively, with a convex shape to extend the surface flashover length both inside and outside. Both the anode and cathode have an on-axis hole of $3 \mathrm{~mm}$ diameter. The hollow cathode cavity was made of stainless steel with outer and inner diameters of 63 and $50 \mathrm{~mm}$, respectively, and was length adjustable. The discharge chamber and external storage capacitor was charged by a $-100 \mathrm{kV}, 40 \mathrm{~mA}$ dc Glassman power supply via a $1.6 \mathrm{M} \Omega$ charging resistor. The amplitude of the charging voltage, which determined the energy of the extracted electron beam, depended on the gas pressure inside the discharge chamber. The external capacitor $(428 \mathrm{pF})$ consisted of three chains of capacitors in parallel to reduce the inductance in the charging circuit. Each chain consisted of seven $15-\mathrm{kV}$, $1000-\mathrm{pF}$ capacitors connected in series. The electron beam was transported and guided from the discharge chamber to the Cherenkov interaction region by the magnetic field of an 


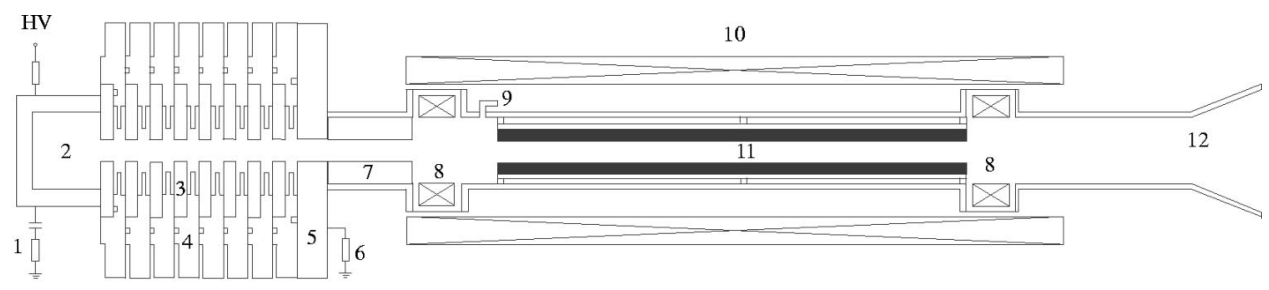

FIG. 1. Cherenkov maser experimental configuration.

1 - Voltage probe 2 - Hollow cathode 3 - Intermediate electrode 4 -Insulator 5 -Anode 6 - CVR

7 - Collimator 8 - Rogowski coil 9 - Gas inlet 10 - Solenoid 11 - Dielectric liner 12 - Waveguide and horn

81-cm long conventional solenoid. The Cherenkov interaction took place in a cylindrical waveguide, $4.75 \mathrm{~mm}$ in radius, lined with a $1.75-\mathrm{mm}$ thick and $60-\mathrm{cm}$ long layer of dielectric (alumina, $\epsilon_{r}=9.5$ ). The microwaves generated by the Cherenkov maser were launched into free space by a well-matched and tapered cylindrical output horn for detailed analysis. The background vacuum requirements were obtained using a pumping system in the form of a two-stage rotary pump and an oil-diffusion pump. The pumping system was located at the output end of the maser connected via a glass "T-piece" chamber. The space around the Cherenkov maser system was shielded by an x-ray protection wall consisting of 7-mm thick lead or 40-cm thick high-density concrete.

In the maser system, the presence of the dielectric in the waveguide reduces the phase velocity of the electromagnetic waves, allowing a resonant interaction to occur between a TM or HE waveguide mode and the rectilinear electron beam. The beam is described as rectilinear because when the beam of $3 \times 10^{-3} \mathrm{~mm}$ in diameter was guided by a magnetic field of $10^{-1} \mathrm{~T}$, its azimuthal velocity was found to be $\sim 10^{6}$ $\mathrm{ms}^{-1}$, which is much smaller than its longitudinal velocity of $1.5 \times 10^{8} \mathrm{~ms}^{-1}$. Coherence of the generated radiation arises due to bunching of electrons in phase with respect to the electromagnetic wave. For values of the beam and waveguide parameters relevant to this experiment, the force exerted on the electrons by the waveguide mode was dominated by the resonant space-charge force, so the maser operated in a Raman-type regime, with strongest amplification of the waveguide mode expected when it was resonant with the slow space-charge wave of the beam, i.e., when the angular frequency, $\omega$, and the axial wave number, $k_{z}$, of the waveguide mode satisfy the relation

$$
\omega \approx k_{z} v_{z}-\frac{\omega_{p}}{\gamma}
$$

where $v_{z}$ is the axial electron velocity, $\gamma=1+|e V| / m_{0} c^{2}$ is the relativistic factor, $\omega_{p}=\sqrt{e^{2} n_{e} / \epsilon_{0} \gamma m_{0}}$ is the plasma frequency, $n_{e}$ is the electron density, $V$ is the beam voltage, and $e$ and $m_{0}$ are the electronic charge and rest mass, respectively. $\omega$ and $k_{z}$ must also, of course, satisfy the characteristic equation for a dielectric-lined cylindrical waveguide, which for a $\mathrm{TM}_{0 n}$ mode is (see the Appendix for the derivation)

$$
\frac{\epsilon \alpha_{2} I_{0}\left(\alpha_{2} a\right)}{\gamma_{1} I_{1}\left(\alpha_{2} a\right)}=\frac{J_{0}\left(\gamma_{1} a\right) Y_{0}\left(\gamma_{1} b\right)-J_{0}\left(\gamma_{1} b\right) Y_{0}\left(\gamma_{1} a\right)}{J_{1}\left(\gamma_{1} a\right) Y_{0}\left(\gamma_{1} b\right)-J_{0}\left(\gamma_{1} b\right) Y_{1}\left(\gamma_{1} a\right)},
$$

where $\gamma_{1}^{2}=\epsilon \omega^{2} / c^{2}-k_{z}^{2}, \alpha_{2}^{2}=k_{z}^{2}-\omega^{2} / c^{2}, a$ is the inner radius of the dielectric, $b$ is the outer radius of the dielectric, $\epsilon$ is the dielectric constant of the liner, and $J_{m}(x), Y_{m}(x)$ and $I_{m}(x)$ are $m$ th-order Bessel functions of the first kind, the second kind, and modified Bessel functions of the first kind, respectively. Figure 2 shows the slow space-charge mode of the electron beam and the $\mathrm{TM}_{01}$ and $\mathrm{TM}_{02}$ waveguide modes for this Cherenkov maser experiment. From this diagram, a resonant interaction would be expected to occur around 21 $\mathrm{GHz}$ with the $\mathrm{TM}_{01}$ mode and around $55 \mathrm{GHz}$ with the $\mathrm{TM}_{02}$ mode.

The vacuum pressure and gas pressure were measured $110 \mathrm{~cm}$ away from the anode near the vacuum pump port and at the anode, respectively, by two active Pirani gauge heads and recorded on a two-channel digital display. The charging voltage was measured by an instantaneous capacitive voltage probe. The discharge current was monitored by an in-line current viewing resistor (CVR) of resistance $0.066 \Omega$.

Fast electron beam diagnostics were designed, constructed, and calibrated, having response times typically of a few ns, which permitted time-correlated observation of the evolution of the electron pulse parameters. The electron beam parameters measured included the beam current (using a compact Rogowski coil upstream from the interaction region) and the transported beam current (using another compact Rogowski coil downstream from the interaction region).

The microwave radiation generated by the Cherenkov maser was launched by a tapered cylindrical output horn for detailed analysis. The microwave pulse duration and temporal profile from the PS-based Cherenkov maser amplifier were obtained by monitoring the output of a rectifying crystal detector with an oscilloscope. The basic microwave measurement system, screened in a metal box, was composed of

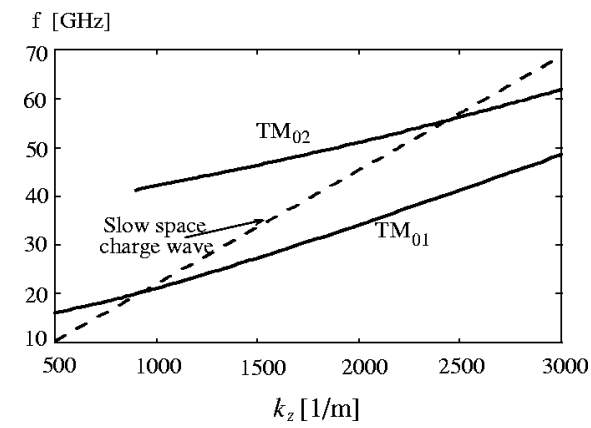

FIG. 2. Dispersion diagram showing the waveguide modes and slow space charge mode of the electron beam $(75 \mathrm{kV}, 10 \mathrm{~A})$. 
Ka-band (26.5-40 GHz) or W-band (75-100 GHz) components starting with a small microwave receiving horn followed by a directional coupler, attenuators, waveguide, and crystal detectors. Two methods were used to identify the frequency range of the Cherenkov radiation. One technique used a series of in-waveguide cutoff filters, and the other used an interferometer. From the first method both the frequency and the energy distribution were obtained. The interferometer was used to further confirm the frequency of the maser output.

The radiation mode pattern (i.e., the field distribution and the power distribution) measurements of the Cherenkov maser were conducted by scanning a radially polarized detector in a circular path centered on the aperture of the output horn while repeatedly pulsing the maser device. The field distribution and the power distribution are dependent on the distance from the launching antenna to the detector, which can be divided into three identifiable regions without sharp boundaries including the reactive near-field region (Rayleigh field), the radiating near-field region (Fresnel region), and the far-field region (Fraunhofer region). The power distribution measurements were conducted in the far-field region. For the Cherenkov maser, the diameter of the output horn was $6 \mathrm{~cm}$ and the dominant frequency of the source was about $25.5 \mathrm{GHz}(\lambda=1.18 \mathrm{~cm})$ and the far-field conditions required that the launching and receiving antennas should be separated by larger than $61 \mathrm{~cm}$. Therefore, the detector was placed $1 \mathrm{~m}$ away from the launching horn. A reference detector system was detached and placed at a fixed position through the entire mode scanning experiment to compensate for random fluctuations in the maser output.

\section{EXPERIMENTAL RESULTS}

The pseudospark (PS) discharge process was studied by monitoring the time-resolved voltage and current. The measured time-correlated PS discharge voltage and current displayed the three stages of a pseudospark discharge: (1) Townsend discharge; (2) hollow cathode discharge; and (3) conductive pseudospark discharge. The Townsend discharge in the PS gives rise to a plasma, i.e., a virtual anode, extending from the anode toward the cathode region. After entering the hollow cathode region, the plasma, with almost the full anode potential, forms a high field sheath surrounding the hollow cathode surface and gives rise to a very fast increase in the emission current. A high current axial electron beam, consisting mainly of electrons from the hollow cathode region behind the cathode hole, is formed during this hollow cathode discharge stage. Afterward, the discharge transfers to the final conductive phase, in which the cold cathode surface facing the anode takes over the whole discharge current with a very high current produced at a low acceleration potential until the gap is shorted. ${ }^{18-20}$

The upper part of Fig. 3 shows a typical record of the time-correlated PS discharge voltage and beam current. The beam current was recorded by a Rowgowski coil located 6 $\mathrm{cm}$ away from the pseudospark anode as shown in Fig. 1. A collimator of $3 \mathrm{~mm}$ aperture and $6 \mathrm{~m}$ in length separated the pseudospark anode from the beam current monitor and was

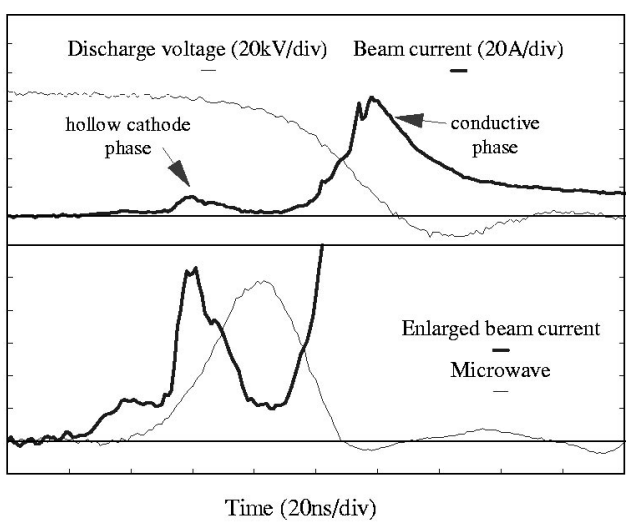

FIG. 3. Typical waveforms of pseudospark discharge voltage, the beam current, and the microwave pulse.

present throughout all the experiments. The duration of the hollow cathode phase of the PS discharge was adjustable between 10-50 ns. The electron beam extracted in the hollow cathode stage had a current of 10-100 A with an acceleration potential close to the applied voltage. The electron beam from the conductive PS discharge had a current of 100-1000 A with beam energy of a few hundred eV.

To obtain a stable, high-quality electron beam, essential for coherent microwave generation, care must be taken with appropriate gas feed and pump points and circuit parameters such as the external capacitance value, etc. Also, in order to achieve the PS hollow cathode effects our experiments showed that (1) the cathode hole diameter should be chosen to be in the range of the cathode thickness $(\sim 3 \mathrm{~mm})$; and (2) the hollow cathode cavity depth should have a minimum value equivalent to the thickness of the cathode.

The operation of the whole maser system is described as follows: The maser system was originally evacuated down to 2-3 mtorr at the discharge chamber and $\sim 0.01 \mathrm{mtorr}$ at the pumping port. The pumping port was connected at the end of the output horn using a glass T-piece chamber. Argon gas was fed into the system from the anode side at a very slow rate of $1 \mathrm{mtorr} / \mathrm{s}$ through a very fine controlled needle valve until a desired gas pressure was reached and balanced by adjustment of the needle valve. The high voltage applied across the pseudospark chamber was increased slowly until breakdown occurred. To achieve a self-pinched, high current electron beam, it was essential to match the applied discharge voltage and chamber gas pressure so that the discharge operated in the pseudospark regime. Our experiments showed an empirical relation of $V_{B}=(0.20 \pm 0.05)$ $\times p^{-4.02 \pm 0.18} d^{-1.77 \pm 0.01}$, where $V_{B}$ is the breakdown voltage of the pseudospark discharge in $\mathrm{kV}$, and $p$ and $d$ are the pressure in torr and the cathode-anode separation in centimeters, respectively. The self-pinched electron beam was extracted from the anode hole of the 8-gap pseudospark discharge chamber just before breakdown occurred and then guided along the beam tube to the Cherenkov interaction region, where the beam interacted with the modes of the dielectric lined waveguide, and the resonant wave was amplified to a few kilowatts from a low power level of a few watts. The microwave radiation generated in the interaction 
region was guided a further $60 \mathrm{~cm}$ in a cylindrical vacuum waveguide before being launched into free space by the conical output horn. The microwave radiation was then diagnosed by a screened and calibrated microwave diagnostic system situated in the far field of the antenna.

Microwave radiation was detected successfully from this first pseudospark-based dielectric Cherenkov maser amplifier. The temporal profile of the microwave output radiation from the maser is shown in the lower part of Fig. 3, time correlated with the electron-beam current and voltage profiles. It was found experimentally that significant microwave radiation was generated only when the dielectric was present in the interaction space, although if there was no dielectric in the cylindrical waveguide, then a very small microwave emission was detected. It was also found that the microwave signal was independent of the guide magnetic field over the range 0.13 to $0.26 \mathrm{~T}$, which ensured that the interaction did not depend on the cyclotron frequency of the electrons. Further frequency measurements and the scanned mode profile $\mathrm{TM}_{01}$ were consistent with the simulations, showing strong coupling with the correct mode for a Cherenkov interaction. In addition, two components of the microwave pulse were observed corresponding to the two energy components of the electron beam during the pseudospark discharge breakdown. Another interesting result was the discovery that the small microwave emission was always present even without the guide B-field or dielectric lining in the waveguide. These results demonstrated that the maser magnification grew from the emission from the PS discharge itself. ${ }^{14,15}$

The frequency range of the microwave radiation from the Cherenkov maser amplifier was measured by applying different cylindrical cutoff filters in the waveguide and comparing this signal with a signal from a nonfiltered reference detector. Using this method, both the Cherenkov maser output and the emission from the PS discharge were analyzed. Figure 4 shows the results with cutoff filters for both the emission from the PS discharge and the Cherenkov maser output signal when the applied discharge voltage was fixed at $\sim 75 \mathrm{kV}$. The waveforms in Fig. 4 were acquired by a digitizing oscilloscope in an averaging mode of 5 shots. The results showed that the emission from the PS discharge had a wide frequency distribution but below the W-band (62.5 $\mathrm{GHz}$ ) cutoff frequency. In the presence of the dielectric, the microwave output had typically two frequency components: one was between 25 and $28.6 \mathrm{GHz}$, and the other between 28.6 and $41.8 \mathrm{GHz}$. By normalizing the Cherenkov maser output signals in Fig. 4, an averaged, normalized microwave output was obtained for each cutoff filter configuration and is shown in Fig. 5. By comparing the time evolution of the beam voltage with the averaged maser output spectrum, it was found that the lower frequency microwave signal corresponded to a $70-80 \mathrm{keV}$ beam energy from the hollow cathode discharge regime and the higher frequency microwave signal to the lower energy beam from the conductive phase of the pseudospark discharge. This increase in frequency with decreasing beam energy is consistent with a Cherenkov interaction mechanism.

An additional method for frequency analysis in the Cherenkov maser was the use of an interferometer. The interfer-
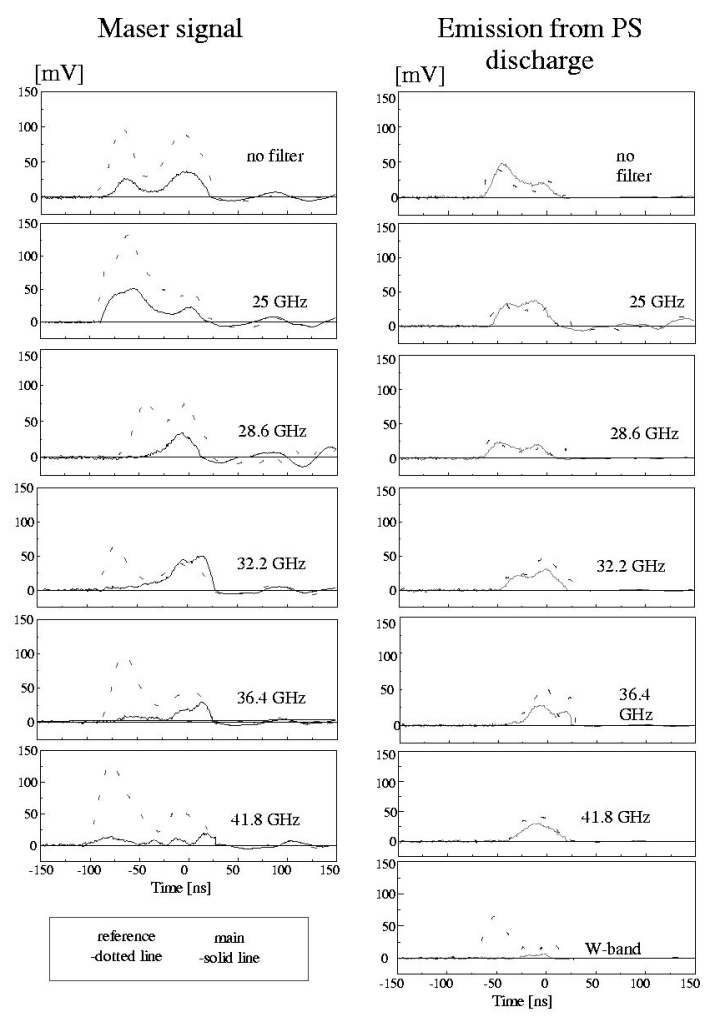

FIG. 4. The results with cutoff filters for both the emissions from the PS and the Cherenkov maser output signal.

ometer in a detecting box consisted of a specially made, T-shaped $K_{a}$-band rectangular waveguide junction with a back-stop adjustor. The movement of the back-stop adjustor changed the phase of the standing wave structure in the waveguide, hence giving rise to a periodic variation of the output amplitude from the detector. The period of the output is expected to be $\lambda_{g} / 2$. Here, $\lambda_{g} / 2$ is the in-guide wavelength of the wave to be measured. The wave frequency can then be calculated by using the following equations:

$$
\begin{aligned}
& f=\frac{\omega}{2 \pi}=\frac{k c}{2 \pi}=\frac{c}{2 \pi} \sqrt{k_{\perp}^{2}+k_{\|}^{2}}, \\
& k_{\perp}=\frac{\pi}{a} ; \quad k_{\|}=\frac{2 \pi}{\lambda_{g}},
\end{aligned}
$$

where $a$ is the larger dimension of the rectangular waveguide. For a $K_{a}$ band waveguide $a$ is equal to $7.112 \mathrm{~mm}$. In

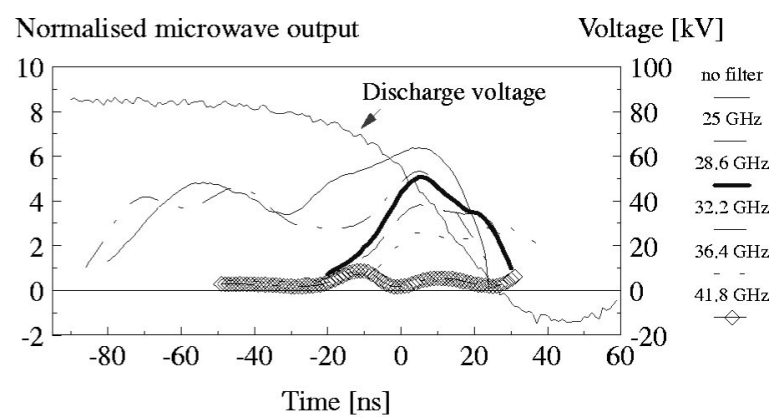

FIG. 5. Normalized Cherenkov maser output signals with various high-pass filters and the time-correlated accelerating potential. 


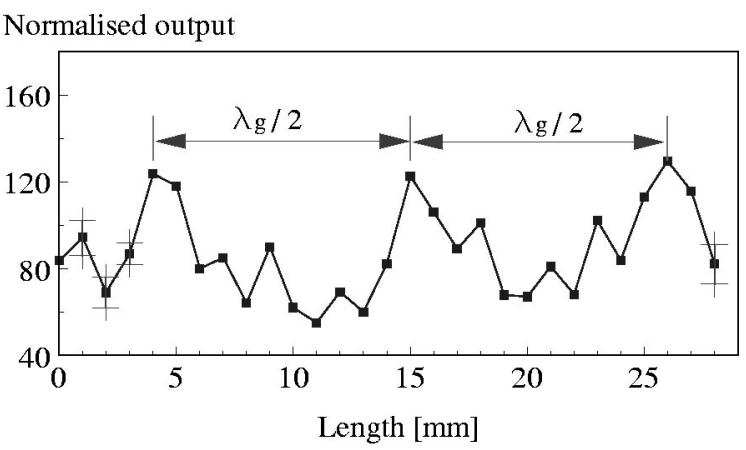

FIG. 6. Frequency identification by an interferometer.

the experiment, to reduce shot-to-shot instabilities of the measurement, a reference signal was recorded and used to normalize the main signal in each position of the back-stop adjustor; five normalized signals were averaged. A typical measurement result at an applied potential of $\sim 75 \mathrm{kV}$ was analyzed against the length of the back-stop adjustor and is shown in Fig. 6. From Fig. 6, $\lambda_{g}$ was measured to be $\sim 21$ $\mathrm{mm}$, corresponding to a frequency of $25.5 \mathrm{GHz}$. This result further confirmed that the radiation has spatial coherence.

To verify the mode of operation as $\mathrm{TM}_{01}$, the far-field output radiation pattern from the conical output horn which had an aperture diameter of $60 \mathrm{~mm}$ was measured. The output antenna pattern associated with the azimuthal E-field component was measured to be independent of the presence of the dielectric and close to zero, confirming the operation of a TM mode. Figure 7 shows the measured pattern associated with the radial E-field component of the radiation with a relative error of $4 \%$ and a systematic error in the radial angle measurement of $<2^{\circ}$. The measured pattern was in good agreement with the results from bench experiments in which a $27 \mathrm{GHz} \mathrm{TM}_{01}$ microwave signal was launched using the same horn. Fraunhofer diffraction theory predicts a maximum at $7.5^{\circ}$, which is in close agreement with both the Cherenkov maser experiment and bench measurements.

In order to calculate the gain of the maser amplifier, both the input power and output power of the maser amplifier were measured. The input power and output power were obtained by integrating the power densities over space. The integrations were completed by numerically integrating the

Normalized microwave output ( $\mathrm{E}_{\mathrm{r}}$ component)

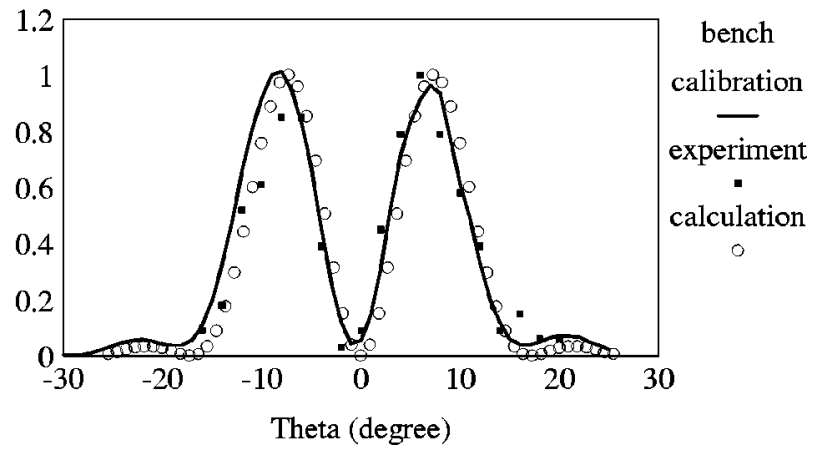

FIG. 7. Far-field mode pattern scan of the radial component $\mathrm{E}_{r}$ compared with calculations and bench calibration. normalized mode profiles and multiplying with the measured maximum power density. In the experiment, there was no external input seed signal from an external microwave radiation source. The microwave emission from the PS discharge acted as the input signal to the amplifier, i.e., the input signal to the Cherenkov maser was generated by the pseudospark discharge itself and was consistent with experiments conducted by Liou et al. ${ }^{14}$ and Ramaswamy et al., ${ }^{15}$ who observed a similar broadband low-power microwave signal from a pseudospark discharge. As shown in Fig. 5, the emission from the PS discharge had a much wider frequency range $(22-50 \mathrm{GHz})$ than the maser output (mainly between 25-28.6 GHz). The emission from the PS discharge was also scanned and found to have similar symmetric mode profiles in both $E_{\phi}$ and $E_{r}$. The total power of the emission from the PS discharge was calculated to be $93 \pm 25 \mathrm{~W}$ in the whole frequency range of $22-50 \mathrm{GHz}$. In the frequency range of 25-28.6 GHz, the fraction of the emission power from the PS was measured to be approximately $(2.7 \pm 0.6) \%$ using a series of waveguide cutoff filters (an example is shown in Fig. 4), resulting in an input power of $2.7 \pm 1.3 \mathrm{~W}$ to the maser at the frequency of maser amplification. With the dielectric present the maser signal was scanned and found to have a $\mathrm{TM}_{01}$ profile. The power of the maser signal was measured using a method similar to that for the background signal. Power from the Cherenkov maser amplifier of 2.0 $\pm 0.2 \mathrm{~kW}$ was measured without considering any losses within the detecting system. The gain of the maser was therefore calculated to be $29 \pm 3 \mathrm{~dB}$.

The observed and measured frequency was found to be $20 \%$ higher than that predicted by the resonance condition for 70-80 keV beam energy in Fig. 2. This discrepancy is probably due to charging of the dielectric liner. ${ }^{13}$ A relative spectral energy distribution was obtained and approximately $65 \%$ of the radiation was found to lie in the $25-28.6 \mathrm{GHz}$ frequency band.

\section{SIMULATIONS}

\section{A. Introduction}

To complement the experimental investigations of the Cherenkov maser, a three-dimensional numerical simulation code was developed. The code integrates the Lorentz equations of motion for the electrons self-consistently with Maxwell's wave equation for a single $\mathrm{TM}_{0 n}$ waveguide mode in the steady-state limit where the relative slippage of the radiation with respect to the electron beam is neglected, as in Ref. 21. It includes the effect of resonant space-charge forces and electron velocity spread.

\section{B. Electromagnetic field evolution}

We now derive equations which describe the evolution of the amplitude and phase of a $\mathrm{TM}_{0 n}$ mode due to its interaction with a beam of electrons.

Maxwell's equations in an isotropic medium can be written as

$\nabla \cdot \mathbf{D}=\rho$, 


$$
\begin{aligned}
& \nabla \cdot \mathbf{B}=0, \\
& \nabla \times \mathbf{E}=-\frac{\partial \mathbf{B}}{\partial t}, \\
& \nabla \times \mathbf{H}=\mathbf{J}+\frac{\partial \mathbf{D}}{\partial t} .
\end{aligned}
$$

Taking the curl of (8), using the vector identity

$$
\nabla \times(\nabla \times \mathbf{A})=\nabla(\nabla \cdot \mathbf{A})-\nabla^{2} \mathbf{A},
$$

and applying (6) and (7), we obtain

$$
\left(\nabla^{2}-\frac{1}{c^{2}} \frac{\partial^{2}}{\partial t^{2}}\right) \mathbf{H}=-\nabla \times \mathbf{J}
$$

We assume that the electric and magnetic fields of the waveguide mode, $\mathbf{E}^{\mathrm{rad}}$ and $\mathbf{H}^{\mathrm{rad}}$, are of the form given by (A2) and (A3) (see the Appendix), with the exception that the complex mode amplitude is now a slowly varying function of space and time, i.e., $G=G(z, t)$, where

$$
\left|\frac{\partial G}{\partial t}\right| \ll \omega|G| \quad \text { and } \quad\left|\frac{\partial G}{\partial z}\right| \ll k|G| \text {, }
$$

and that the transverse structure of the modes as described by $\mathbf{e}$ and $\mathbf{h}$ is unaffected by the presence of the electron beam. This allows (9) to be written as

$$
\left(\frac{\partial^{2}}{\partial z^{2}}-\frac{1}{c^{2}} \frac{\partial^{2}}{\partial t^{2}}-\kappa^{2}\right) \mathbf{H}^{\mathrm{rad}}=-\nabla \times \mathbf{J} .
$$

As $\mathbf{H}^{\mathrm{rad}}=H_{\theta} \hat{\theta}$ for $\mathrm{TM}_{0 n}$ modes, we can write

$$
\begin{aligned}
& \frac{1}{2}\left(\frac{\partial^{2}}{\partial z^{2}}-\frac{1}{c^{2}} \frac{\partial^{2}}{\partial t^{2}}-\kappa^{2}\right)\left(G(z, t) e^{i(k z-\omega t)}\right) h_{\theta}+\text { c.c. } \\
& \quad=\frac{\partial J_{z}}{\partial r}-\frac{\partial J_{r}}{\partial z}
\end{aligned}
$$

where we have again assumed azimuthal symmetry, i.e., $\partial / \partial \theta=0$. Using the slowly varying envelope approximation

$$
\begin{aligned}
& \left(\frac{\partial^{2}}{\partial z^{2}}-\frac{1}{c^{2}} \frac{\partial^{2}}{\partial t^{2}}-\kappa^{2}\right)\left(G(z, t) e^{i(k z-\omega t)}\right) \\
& \quad \approx 2 i k\left(\frac{\partial G}{\partial z}+\frac{\omega}{c^{2} k} \frac{\partial G}{\partial t}\right) e^{i(k z-\omega t)}+\left(\frac{\omega^{2}}{c^{2}}-k^{2}\right) G e^{i(k z-\omega t)},
\end{aligned}
$$

(10) becomes

$$
i k\left(\frac{\partial G}{\partial z}+\frac{\omega}{c^{2} k} \frac{\partial G}{\partial t}\right) h_{\theta} e^{i(k z-\omega t)}+\text { c.c. }=\left(\frac{\partial J_{z}}{\partial r}-\frac{\partial J_{r}}{\partial z}\right) .
$$

As we have assumed azimuthal symmetry, the current and charge densities driving the electromagnetic field will therefore correspond to those of thin charged rings, as shown in Fig. 8.

The form of the current density $\mathbf{J}$ can be deduced from Fig. 8. Each electron represents a ring of charge $q$ with $|q|$ $\gg|e|$, i.e.,

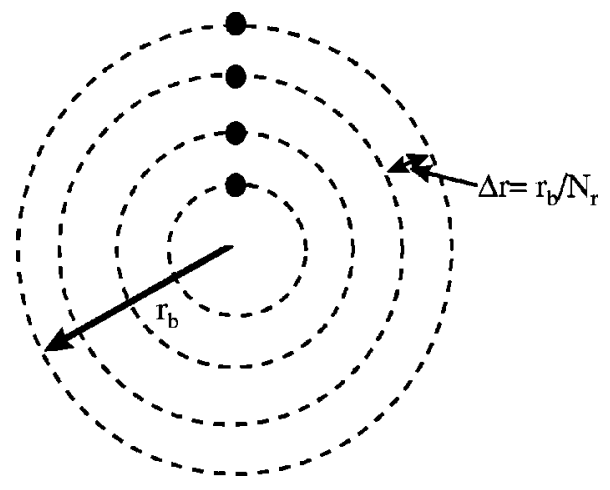

FIG. 8. Representation of the electron beam in the 3D model.

$$
\mathbf{J}=-\sum_{j=1}^{N} q_{j} \frac{\delta\left(r-r_{j}\right)}{2 \pi r} \delta\left(z-z_{j}\right),
$$

where $q_{j}=2 \pi r_{0 j} \lambda, r_{0 j}$ is the initial radius of each ring and $\lambda$ is the linear charge density of each ring. $\lambda$ can be determined from the fact that in our steady-state model, $N$ rings cross a fixed point $z$ in one wave period, so that

$$
I=\sum_{j=1}^{N} q_{j} \frac{\omega}{2 \pi}=\lambda \omega \sum_{j=1}^{N} r_{0 j} .
$$

After evaluating the sum, this can be rearranged to give $\lambda$ and consequently $q_{j}$

$$
q_{j}=\frac{4 \pi r_{0 j} I}{\omega N r_{b}},
$$

where $r_{b}$ is the electron beam radius. Defining $\widetilde{\mathbf{J}}$ as

$$
\widetilde{\mathbf{J}}=\frac{1}{\pi} \int_{0}^{2 \pi} \mathbf{J} e^{-i(k z-\omega t)} d(\omega t),
$$

then

$$
\begin{aligned}
& \widetilde{J}_{z}=-\frac{\omega}{\pi} \sum_{j=1}^{N} q_{j} \frac{\delta\left(r-r_{j}\right)}{2 \pi r} e^{-i\left(k z-\omega t_{j}\right)}, \\
& \widetilde{J}_{r}=-\frac{\omega}{\pi} \sum_{j=1}^{N} q_{j} \frac{v_{r j}}{v_{z j}} \frac{\delta\left(r-r_{j}\right)}{2 \pi r} e^{-i\left(k z-\omega t_{j}\right)},
\end{aligned}
$$

and Eq. (11) becomes

$$
i k \frac{d G}{d z} h_{\theta} e^{i(k z-\omega t)} \approx\left(\frac{\partial \widetilde{J}_{z}}{\partial r}-i k \widetilde{J}_{r}\right),
$$

where we have averaged over a wave period and dropped the time derivative of $G$, i.e., we assume steady-state amplification, so $G=G(z)$. Substituting (14) and (15) in (16), we obtain

$$
\begin{aligned}
\frac{d G}{d z} h_{\theta}= & \frac{i \omega}{2 \pi k} \sum_{j=1}^{N} q_{j}\left(\frac{\delta^{\prime}\left(r-r_{j}\right)}{2 \pi r}-\frac{\delta\left(r-r_{j}\right)}{2 \pi r^{2}}\right. \\
& \left.-i k \frac{v_{r j}}{v_{z j}} \frac{\delta\left(r-r_{j}\right)}{2 \pi r}\right) e^{-i\left(k z-\omega t_{j}\right)} .
\end{aligned}
$$

Multiplying both sides by $e_{r}^{*}$ and integrating over the entire waveguide cross section gives 


$$
\begin{aligned}
\left(2 \pi \int h_{\theta} e_{r}^{*} r d r\right) \frac{d G}{d z} \\
=\frac{i \omega}{2 \pi k} \sum_{j=1}^{N} q_{j} e^{-i\left(k z-\omega t_{j}\right)} \int\left(\delta^{\prime}\left(r-r_{j}\right)-\frac{\delta\left(r-r_{j}\right)}{r}\right. \\
\left.\quad-i k \frac{v_{r j}}{v_{z j}} \delta\left(r-r_{j}\right)\right) e_{r}^{*} d r .
\end{aligned}
$$

Now

$$
2 \pi \int h_{\theta} e_{r}^{*} r d r=\omega k \epsilon_{0} Q
$$

where $Q$ is the mode-dependent constant defined in (A18). Therefore, (17) reduces to

$$
\begin{aligned}
\frac{d G}{d z}= & \frac{i}{2 \pi k^{2} \epsilon_{0} Q} \sum_{j=1}^{N} q_{j} e^{-i\left(k z-\omega t_{j}\right)} \\
& \times \int\left(\delta^{\prime}\left(r-r_{j}\right)-\frac{\delta\left(r-r_{j}\right)}{r}-i k \frac{v_{r j}}{v_{z j}} \delta\left(r-r_{j}\right)\right) e_{r}^{*} d r .
\end{aligned}
$$

Consider the integral on the right-hand side (RHS) of (18). Using the fact that

$$
e_{r}=\frac{-i k}{\alpha_{2}^{2}} \frac{d e_{z 2}}{d r}=\frac{-i k}{\alpha_{2}^{2}} I_{0}^{\prime}\left(\alpha_{2} r\right),
$$

then

$$
\int e_{r}^{*}(r) \delta^{\prime}\left(r-r_{j}\right) d r=-\left.\frac{d e_{r}^{*}}{d r}\right|_{r=r_{j}}=-i k I_{0}^{\prime \prime}\left(\alpha_{2} r\right) .
$$

Now, $y=I_{0}(x)$ satisfies the modified Bessel equation

$$
\frac{d^{2} y}{d x^{2}}+\frac{1}{x} \frac{d y}{d x}-y=0,
$$

so solving for $d^{2} y / d x^{2}$ it can be shown that

$$
\int e_{r}^{*}(r) \delta^{\prime}\left(r-r_{j}\right) d r=i k\left(\frac{I_{1}\left(\alpha_{2} r_{j}\right)}{\alpha_{2} r_{j}}-I_{0}\left(\alpha_{2} r_{j}\right)\right) .
$$

It is now straightforward to show that the integral on the RHS of (18) reduces to

$$
\begin{gathered}
\int\left(\delta^{\prime}\left(r-r_{j}\right)-\frac{\delta\left(r-r_{j}\right)}{r}-i k \frac{v_{r j}}{v_{z j}} \delta\left(r-r_{j}\right)\right) e_{r}^{*} d r \\
=-i k\left(I_{0}\left(\alpha_{2} r_{j}\right)+i \frac{k}{\alpha_{2}} v_{r j} v_{z j} I_{1}\left(\alpha_{2} r_{j}\right)\right),
\end{gathered}
$$

so that (18) becomes

$$
\begin{aligned}
\frac{d G}{d z}= & \frac{1}{2 \pi k \epsilon_{0} Q} \sum_{j=1}^{N} q_{j} e^{-i\left(k z-\omega t_{j}\right)}\left(I_{0}\left(\alpha_{2} r_{j}\right)\right. \\
& \left.+i \frac{k}{\alpha_{2}} v_{r j} v_{z j} I_{1}\left(\alpha_{2} r_{j}\right)\right) .
\end{aligned}
$$

Substituting for $q_{j}$ using (13) gives

$$
\begin{aligned}
\frac{d G}{d z}= & \frac{2 I}{k \omega \epsilon_{0} r_{b} Q}\left\langle r_{0 j}\left(I_{0}\left(\alpha_{2} r_{j}\right)+\frac{i k}{\alpha_{2}} \frac{v_{r j}}{v_{z j}} I_{1}\left(\alpha_{2} r_{j}\right)\right)\right. \\
& \left.\times e^{-i\left(k z-\omega t_{j}\right)}\right\rangle,
\end{aligned}
$$

where $\langle\ldots\rangle$ is an average over the entire electron ensemble. This equation describes the evolution of the complex amplitude of a $\mathrm{TM}_{0 n}$ mode due to its interaction with a beam of electrons.

\section{Space charge}

In addition to the radiation fields of the waveguide mode, the electrons will also experience forces due to spacecharge fields. The analytical description of space charge forces in two or three dimensions is in general complicated and demanding in terms of the computer time and memory required. In this section we derive expressions for the resonant ac space-charge fields using a simplified model.

As in the previous sections, we assume azimuthal symmetry. The charge density is therefore of the form

$$
\begin{aligned}
\rho & =-\sum_{j=1}^{N} q_{j} \frac{\delta\left(r-r_{j}\right)}{2 \pi r} \delta\left(z-z_{j}\right) \\
& =-\omega \sum_{j=1}^{N} q_{j} \frac{\delta\left(r-r_{j}\right)}{2 \pi r} \delta\left(\omega t-\omega t_{j}\right) .
\end{aligned}
$$

Assuming that the charge density can be written as

$$
\rho=\frac{1}{2}\left(\tilde{\rho} e^{i(k z-\omega t)}+\text { c.c. }\right),
$$

multiplying both sides by $e^{-i(k z-\omega t)}$ and integrating over $\omega t$ from $0 \rightarrow 2 \pi$ gives

$$
\begin{aligned}
\tilde{\rho} & =\frac{1}{\pi} \int_{0}^{2 \pi} \rho e^{-i(k z-\omega t)} d(\omega t) \\
& =\frac{\omega}{\pi} \sum_{j=1}^{N} q_{j} \frac{\delta\left(r-r_{j}\right)}{2 \pi r} \frac{e^{-i(k z-\omega t)}}{v_{z j}} .
\end{aligned}
$$

From Gauss's law,

$$
\nabla \cdot \mathbf{E}^{\mathrm{sc}}=\frac{\rho}{\epsilon_{0}}
$$

so writing $\mathbf{E}^{\mathrm{sc}}$ as

$$
\mathbf{E}^{\mathrm{sc}}=\frac{1}{2} \widetilde{\mathbf{E}}^{\mathrm{sc}} e^{i(k z-\omega t)}+\text { c.c. },
$$

and assuming that the space-charge field acts only in the $z$ direction, i.e., $\mathbf{E}^{\mathrm{sc}}=E^{\mathrm{sc}} \hat{\mathbf{z}}$, then it can be shown, using (21), that

$$
\widetilde{E}^{\mathrm{sc}}=-i \frac{\tilde{\rho}}{k \epsilon_{0}}=\frac{i \omega}{k \epsilon \pi} \sum_{j} q_{j} \frac{\delta\left(r-r_{j}\right)}{2 \pi r} \frac{e^{-i(k z-\omega t)}}{v_{z j}} .
$$

Note from (22) that $\widetilde{E}^{\text {sc }}$ is infinite at $r=r_{j}$. In order to remove this singularity we average $\widetilde{E}^{\mathrm{sc}}$ over a small area. To do this, we again consider the transverse cross section of the electron beam as being divided into $N_{r}$ rings (Fig. 9). For the purposes of the space-charge calculation, however, we con- 


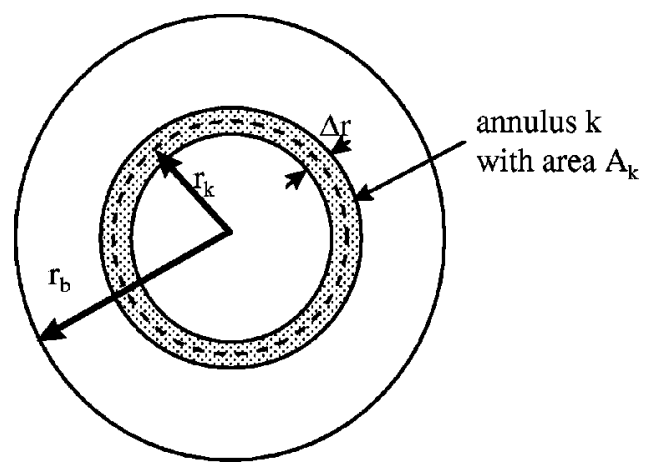

FIG. 9. Diagram of space-charge model.

sider these rings as being the centers of $N_{r}$ annuli, each of equal thickness $\Delta r=r_{b} / N_{r}$. The central radius of the $k$ th annulus is

$$
r_{k}=\left(k-\frac{1}{2}\right) \Delta r
$$

initially, so that $k=1$ is the innermost annulus, and $k=N_{r}$ is the outermost annulus. The initial area of the $j$ th annulus is given by

$$
A_{k}=\pi\left(r_{k}+\frac{\Delta r}{2}\right)^{2}-\pi\left(r_{k}-\frac{\Delta r}{2}\right)^{2}=2 \pi r_{k} \Delta r .
$$

We will average the space-charge force over the crosssectional area of each annulus $A_{k}$ so

$$
\begin{aligned}
\overline{E_{k}^{\mathrm{sc}}} & =\frac{1}{A_{k}} 2 \pi \int_{r_{k}-\Delta r / 2}^{r_{k}+\Delta r / 2} E^{\mathrm{sc}} r d r \\
& =\frac{i \omega}{2 \pi k \epsilon_{0} A_{k}} \sum_{j=1}^{N_{k}} q_{j} \frac{e^{-i(k z-\omega t)}}{v_{z j}}+\text { c.c. }
\end{aligned}
$$

where $N_{k}$ is the number of rings lying in annulus $k$, i.e., with $r_{k}-\Delta r / 2<r_{j}<r_{k}+\Delta r / 2$. Using the definition of $q_{j}$ in (13) and the fact that in a given annulus $k, r_{o j}=r_{k}$ for all $j$ $=1, \ldots, N_{k}$, this then becomes

$$
\overline{E_{k}^{\mathrm{sc}}}=\frac{i I N_{r}}{k \epsilon_{0} \pi r_{b}^{2} N} \sum_{j=1}^{N_{k}} \frac{e^{-i(k z-\omega t)}}{v_{z j}}+\text { c.c. }
$$

Note that if all electrons remain within a distance $\Delta r / 2$ of their initial radial position, then $N_{k}=N / N_{r}$ always, and (24) takes the particularly simple form

$$
\overline{E_{k}^{\mathrm{sc}}}=\frac{i I}{k \epsilon_{0} \pi r_{b}^{2}}\left\langle\frac{e^{-i \phi}}{v_{z}}\right\rangle_{k}+\text { c.c. },
$$

where $\langle\ldots\rangle_{k}$ is an average over the electrons within the $k$ th annulus. Note that the space-charge field in one annulus acts only on the electrons within that same annulus and has no influence on the electrons in other annuli. Under most conditions, (24) and (25) should be almost equivalent. A case where (25) would not be a good approximation would be when there is a strong dc space-charge field, and a weak guide magnetic field. In this case, the beam will spread radially and the number of electrons in each annulus will change substantially from their initial values. For the parameters in this paper, the effect of dc space-charge fields are dominated by that of the guide field and can be neglected.

\section{Electron dynamics}

In order to describe the interaction between the electron beam and the $\mathrm{TM}_{0 n}$ waveguide mode self-consistently, it is necessary to describe the motion of the electrons under the action of the fields of the waveguide mode, the space-charge fields, and the guide magnetic field $\mathbf{B}_{0}$. The force on the $j$ th charged ring making up the electron beam is determined by the Lorentz equation

$$
\mathbf{F}_{j}=-q_{j}\left(\mathbf{E}+\mathbf{v}_{j} \times \mathbf{B}\right),
$$

where $\mathbf{v}_{j}$ is the velocity of each electron comprising the ring and the electric field $\mathbf{E}$ is a superposition of the radiation electric fields, $\mathbf{E}^{\mathrm{rad}}$, as given by (A2) and (A9), and the ac space-charge field, $\mathbf{E}^{\mathrm{sc}}$, as given by (25). The magnetic field $\mathbf{B}$ is a superposition of the radiation fields, $\mathbf{B}^{\mathrm{rad}}$, as given by (A3) and (A9), and the guide magnetic field $\mathbf{B}_{0}=B_{0} \hat{\mathbf{z}}$.

This force determines the positions $\left(\mathbf{r}_{j}\right)$ and scaled momenta $\left(\mathbf{u}_{j}=\gamma_{j} \mathbf{v}_{j}\right)$ of the electrons (rings) via

$$
\begin{aligned}
& \frac{d \mathbf{r}_{j}}{d t}=\frac{\mathbf{u}_{j}}{\gamma_{j}} \\
& \frac{d \mathbf{u}_{j}}{d t}=\frac{\mathbf{F}_{j}}{m_{j}}=-\frac{q_{j}}{m_{j}}\left(\mathbf{E}^{\mathrm{rad}}+\mathbf{E}^{\mathrm{sc}}+\frac{\mathbf{u}_{j}}{\gamma_{j}} \times\left(\mathbf{B}^{\mathrm{rad}}+\mathbf{B}_{0}\right)\right),
\end{aligned}
$$

where $\mathbf{u}_{j}=\gamma_{j} \mathbf{v}_{j}, m_{j}=q_{j} m_{e} /|e|$ is the mass of the $j$ th ring, $m_{e}$ is the mass of a single electron, and $\gamma_{j}=(1$ $\left.+\left|\mathbf{u}_{j}\right|^{2} / c^{2}\right)^{1 / 2}$ is the relativistic factor.

As we are describing a steady-state amplifier, it is convenient to change the independent variable from $t$ to $z$, via $d / d t \rightarrow v_{z_{j}} d / d z$ so that (27) and (28) become

$$
\begin{aligned}
& \frac{d t_{j}}{d z}=\frac{\gamma_{j}}{u_{z_{j}}}, \\
& \frac{d r_{j}}{d z}=\frac{u_{r_{j}}}{u_{z_{j}}}, \\
& \frac{d \mathbf{u}_{j}}{d z}=-\frac{|e|}{m_{e} v_{z_{j}}}\left(\mathbf{E}^{\mathrm{rad}}+\mathbf{E}^{\mathrm{sc}}+\frac{\mathbf{u}_{j}}{\gamma_{j}} \times\left(\mathbf{B}^{\mathrm{rad}}+\mathbf{B}_{0}\right)\right) .
\end{aligned}
$$

Equations (29) through (31), in combination with Eqs. (19) and (25), form a closed system of equations which allows a self-consistent description of the Cherenkov interaction between an electron beam and a $\mathrm{TM}_{0 n}$ waveguide mode.

\section{E. Results}

Figure 10 shows a graph of the predicted output power from the Cherenkov maser as a function of interaction length for the $\mathrm{TM}_{01}$ and $\mathrm{TM}_{02}$ modes when the beam energy is 75 $\mathrm{keV}$ and current is $10 \mathrm{~A}$. Only these two modes lie within the frequency range of the radiation emitted by the PS discharge. The microwave power at $z=0$ was assumed to be $3 \mathrm{~W}$ for both modes. The electron beam was assumed to be a perfectly collimated solid beam, with an axial velocity spread $\left(\Delta v_{z} / v_{z}\right)$ of $3 \%$, and a radius of $1.5 \mathrm{~mm}$. It can be seen 


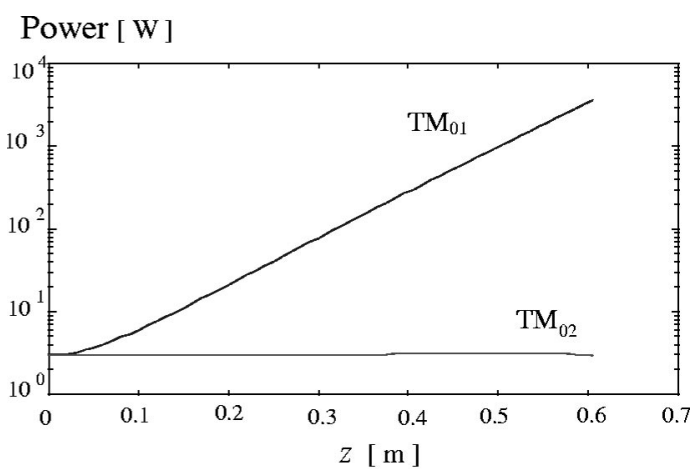

FIG. 10. Calculated output power of $\mathrm{TM}_{01}$ and $\mathrm{TM}_{02}$ modes as a function of $z$.

from Fig. 10 that the $\mathrm{TM}_{01}$ mode at $\sim 21 \mathrm{GHz}$ is amplified strongly, attaining a power of $\sim 3.4 \mathrm{~kW}$ at $z=60 \mathrm{~cm}$, whereas the power in the $\mathrm{TM}_{02}$ mode at $\sim 55 \mathrm{GHz}$ remains around its initial level. These simulations support the interpretation of the experimental results as microwave amplification via a Cherenkov interaction between the high-quality electron beam and the $\mathrm{TM}_{01}$ mode of the dielectric-lined waveguide.

\section{CONCLUSIONS}

In conclusion, we have presented the first measurements of coherent electromagnetic radiation generation in a freeelectron maser using an electron beam from a pseudospark discharge, which were consistent with the results from the simulations showing strong coupling with the correct mode for a Cherenkov interaction. The microwave radiation was generated by Cherenkov amplification of the broadband emission from the pseudospark discharge. A microwave emission of around $100 \mathrm{~W}$ from the PS discharge was measured in the frequency range $20-50 \mathrm{GHz}$ with a percentage of $(2.7 \pm 0.6) \%$ in the frequency range $25.5-28.6 \mathrm{GHz}$. The frequency of the microwave output after the Cherenkov maser interaction was measured to be mainly around $25.5 \mathrm{GHz}$ and the dominating mode was identified as being $\mathrm{TM}_{01}$. The duration of the microwave pulse was approximately $80 \mathrm{~ns}$, with a peak power of around $2 \pm 0.2 \mathrm{~kW}$. The gain of this amplifier was measured as $29 \pm 3 \mathrm{~dB}$. The microwave output was found to be insensitive to the magnitude of the applied magnetic field, which was varied from 0.13 to $0.26 \mathrm{~T}$. The observed frequency, $25.5 \mathrm{GHz}$, was found to be slightly higher than that predicted by the resonance condition for the above beam parameters and the cold alumina-lined waveguide $(21 \mathrm{GHz})$. This discrepancy could be explained as the effect of either the charging of the dielectric liner or the beam loading effect on the transverse structure of the TM waveguide mode.

Future fundamental aims would be to improve the performance of the pseudospark discharge with respect to microwave power, pulse repetition frequency, and further investigation of the transportation of this novel electron beam in the low pressure gas-filled interaction region. This work

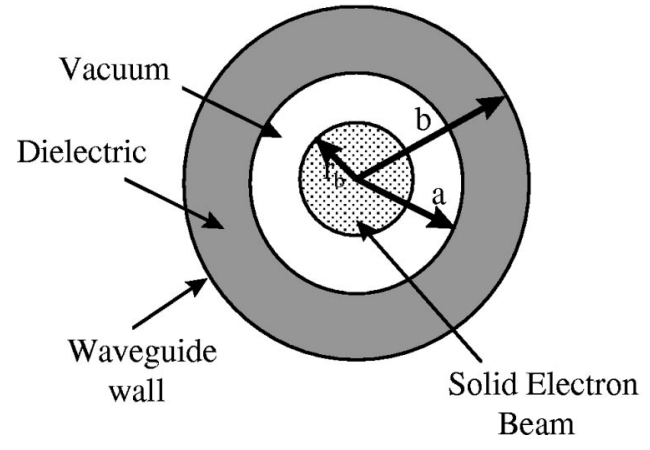

FIG. 11. Diagram of the cross section of a circular dielectric lined waveguide.

could potentially lead to a whole series of novel microwave sources and amplifiers employing a pseudospark-based highdensity, high-brightness electron beam.

\section{ACKNOWLEDGMENTS}

H.Y. would like to thank the Engineering and Physical Sciences Research Council (EPSRC) and the Committee of Vice-Chancellors and Principals of the Universities of the United Kingdom and the University of Strathclyde for financial support so that this work could be started and continue. Assistance from P. Aitken and C. Whyte is also gratefully acknowledged.

\section{APPENDIX: DIELECTRIC WAVEGUIDE MODES}

\section{1. $\mathrm{TM}_{0 n}$ modes}

A diagram of the cross section of a dielectric lined circular waveguide is shown in Fig. 11. In the absence of any charges or currents, Maxwell's wave equation for the electric field is

$$
\left(\nabla^{2}-\frac{1}{c^{2}} \frac{\partial^{2}}{\partial t^{2}}\right) \mathbf{E}^{\mathrm{rad}}=0 .
$$

Let us assume that the radiation electric field in the waveguide is of the form

$$
\mathbf{E}^{\mathrm{rad}}=\frac{1}{2}\left(G e^{i(k z-\omega t)} \mathbf{e}(r, \theta)+\text { c.c. }\right),
$$

and that the magnetic field in the waveguide is of the form

$$
\mathbf{H}^{\mathrm{rad}}=\frac{1}{2}\left(G e^{i(k z-\omega t)} \mathbf{h}(r, \theta)+\text { c.c. }\right) \text {. }
$$

In these expressions, $G$ is a constant complex amplitude and $\mathbf{e}, \mathbf{h}$ are complex mode vectors.

We restrict our analysis to azimuthally symmetric $\mathrm{TM}_{0 n}$ modes. Substituting (A2) into (A1), it can be shown that for $\mathrm{TM}_{0 n}$ modes, the $z$ component of $\mathbf{e}, e_{z}$, satisfies the relation

$$
\left(\nabla_{\perp}^{2}+\left(\mu \epsilon \frac{\omega^{2}}{c^{2}}-k^{2}\right)\right) e_{z}(r)=0 .
$$

As these modes are azimuthally symmetric, i.e., e $=\mathbf{e}(r)$, the transverse derivative in the expression above reduces to 


$$
\nabla_{\perp}^{2}=\frac{\partial^{2}}{\partial r^{2}}+\frac{1}{r} \frac{\partial}{\partial r},
$$

so (A4) becomes

$$
\left(\frac{\partial^{2}}{\partial r^{2}}+\frac{1}{r} \frac{\partial}{\partial r}+\kappa^{2}\right) e_{z}(r)=0
$$

where $\kappa^{2}=\mu \epsilon \omega^{2} / c^{2}-k^{2}$. Equation (A5) is a form of Bessel's equation of order 0 , so $e_{z}$ is a linear combination of zeroth-order Bessel functions, i.e.,

$$
\begin{aligned}
& e_{z 1}=A_{1} J_{0}\left(\kappa_{1} r\right)+B_{1} Y_{0}\left(\kappa_{1} r\right) \quad \text { when } a<r<b, \\
& e_{z 2}=J_{0}\left(\kappa_{2} r\right)+B_{2} Y_{0}\left(\kappa_{2} r\right) \quad \text { when } 0<r<a,
\end{aligned}
$$

where $\kappa_{1,2}^{2}=\mu_{1,2} \epsilon_{1,2} \omega^{2} / c^{2}-k^{2}$ and $A_{1}, B_{1}$, and $B_{2}$ are constants which are determined by the boundary conditions to be shown shortly.

\section{Characteristic equation}

The transverse field components can be obtained from the longitudinal field components (A6) and (A7) using the relations

$$
\begin{aligned}
& \mathbf{h}_{\perp}=\frac{1}{\kappa^{2}}\left(i \omega \epsilon \epsilon_{0} \hat{\mathbf{z}} \times \nabla_{\perp} e_{z}\right), \\
& \mathbf{e}_{\perp}=\frac{1}{\kappa^{2}}\left(i k \nabla_{\perp} e_{z}\right),
\end{aligned}
$$

so that the field components in each region can be written as:

$$
\begin{aligned}
& \text { Region 1: dielectric }(a<r<b) \\
& e_{r 1}=\frac{i k}{\kappa_{1}}\left(A_{1} J_{0}^{\prime}\left(\kappa_{1} r\right)+B_{1} Y_{0}^{\prime}\left(\kappa_{1} r\right)\right), \quad h_{r 1}=0 \\
& e_{\theta 1}=0, \quad h_{\theta 1}=\frac{\omega \epsilon_{0} \epsilon_{1}}{k} e_{r 1} \\
& e_{z 1}=A_{1} J_{0}\left(\kappa_{1} r\right)+B_{1} Y_{0}\left(\kappa_{1} r\right), \quad h_{z 1}=0 ;
\end{aligned}
$$

(ii) Region 2: vacuum $(0<r<b)$

$$
\begin{aligned}
& e_{r 2}=\frac{i k}{\kappa_{2}} J_{0}^{\prime}\left(\kappa_{2} r\right), \quad h_{r 2}=0 \\
& e_{\theta 2}=0, \quad h_{\theta 2}=\frac{\omega \epsilon_{0} \epsilon_{2}}{k} e_{r 2} \\
& e_{z 2}=J_{0}\left(\kappa_{2} r\right), \quad h_{z 2}=0 .
\end{aligned}
$$

As $r \rightarrow 0, Y_{m}\left(\kappa_{2} r\right) \rightarrow \infty$ for all $m$ so $B_{2}=0$ in order that the fields remain finite at all points across the cross section of the waveguide.

In order to determine the arbitrary constants $A_{1}$, and $B_{1}$, we must use the boundary conditions on $\mathbf{E}$ and $\mathbf{H}$, at the boundaries $r=a$ and $r=b$. At $r=a$, the vacuum/dielectric boundary, the continuity of $h_{\theta}$ implies that

$$
\frac{\epsilon_{1}}{\kappa_{1}}\left(A_{1} J_{0}^{\prime}\left(\kappa_{1} a\right)+B_{1} Y_{0}^{\prime}\left(\kappa_{1} a\right)\right)=\frac{\epsilon_{1}}{\kappa_{2}} J_{0}^{\prime}\left(\kappa_{1} a\right),
$$

and the continuity of $e_{z}$ implies that

$$
A_{1} J_{0}\left(\kappa_{1} a\right)+B_{1} Y_{0}\left(\kappa_{1} a\right)=J_{0}\left(\kappa_{2} a\right) .
$$

At $r=b$, the dielectric/perfect conductor boundary, the continuity of $e_{z}$ implies that $e_{z}\left(r=r_{b}\right)=0$, so

$$
B_{1}=-\frac{J_{0}\left(\kappa_{1} b\right)}{Y_{0}\left(\kappa_{1} b\right)} A_{1} .
$$

Substituting (A12) in (A10) implies that

$$
A_{1}=\frac{J_{0}\left(\kappa_{2} a\right) Y_{0}\left(\kappa_{1} b\right)}{J_{0}\left(\kappa_{1} a\right) Y_{0}\left(\kappa_{1} b\right)-J_{0}\left(\kappa_{1} b\right) Y_{0}\left(\kappa_{1} a\right)},
$$

whereas substituting (A12) in (A11) implies that

$$
A_{1}=\frac{\epsilon_{2}}{\epsilon_{1}} \frac{\kappa_{1}}{\kappa_{2}} \frac{J_{0}^{\prime}\left(\kappa_{2} a\right) Y_{0}\left(\kappa_{1} b\right)}{J_{0}^{\prime}\left(\kappa_{1} a\right) Y_{0}\left(\kappa_{1} b\right)-J_{0}\left(\kappa_{1} b\right) Y_{0}^{\prime}\left(\kappa_{1} a\right)} .
$$

For these two expressions to be consistent, it is necessary that

$$
\begin{aligned}
& \epsilon_{1} \kappa_{2} J_{0}\left(\kappa_{2} a\right)\left(J_{0}^{\prime}\left(\kappa_{1} a\right) Y_{0}\left(\kappa_{1} b\right)-J_{0}\left(\kappa_{1} b\right) Y_{0}^{\prime}\left(\kappa_{1} a\right)\right) \\
& \quad=\epsilon_{2} \kappa_{1} J_{0}^{\prime}\left(\kappa_{2} a\right)\left(J_{0}\left(\kappa_{1} a\right) Y_{0}\left(\kappa_{1} b\right)-J_{0}\left(\kappa_{1} b\right) Y_{0}\left(\kappa_{1} a\right)\right)
\end{aligned}
$$

which is the characteristic equation for a $\mathrm{TM}_{0 n}$ mode.

In order to have a resonant interaction between the $\mathrm{TM}_{0 n}$ mode and an electron beam, the phase velocity in region 2 (vacuo) must be less than $c$, so we consider only cases where $\kappa_{2}^{2}<0$. Defining $\kappa_{2}=i \alpha_{2}$, and using the fact that

$$
J_{m}(i x)=i^{m} I_{m}(x),
$$

where $I_{m}(x)$ is a modified Bessel function of the first kind, then the characteristic equation for the $\mathrm{TM}_{0 n}$ modes (A13) becomes

$$
\begin{aligned}
& \epsilon \alpha_{2} I_{0}\left(\alpha_{2} a\right)\left(J_{1}\left(\kappa_{1} a\right) Y_{0}\left(\kappa_{1} b\right)-J_{0}\left(\kappa_{1} b\right) Y_{1}\left(\kappa_{1} a\right)\right) \\
& \quad=\kappa_{1} I_{1}\left(\alpha_{2} a\right)\left(J_{0}\left(\kappa_{1} a\right) Y_{0}\left(\kappa_{1} b\right)-J_{0}\left(\kappa_{1} b\right) Y_{0}\left(\kappa_{1} a\right)\right) .
\end{aligned}
$$

\section{Power}

The flow of electromagnetic power across a surface is determined by the $z$ component of the Poynting vector, defined as

$$
\mathbf{S}=\mathbf{E} \times \mathbf{H},
$$

when integrated over the surface, i.e., waveguide crosssection area. Note that as electromagnetic energy propagates inside the dielectric, it is necessary to integrate over both regions of the dielectric, i.e., from $r=0 \rightarrow b$. The power at a position $z$ is therefore

$$
P=\int_{A}(\mathbf{E} \times \mathbf{H})_{z} d A .
$$

For a $\mathrm{TM}_{0 n}$ mode, $\mathbf{E}=E_{r} \hat{\mathbf{r}}+E_{z} \hat{\mathbf{z}}$ and $\mathbf{H}=H_{\theta} \hat{\theta}$, so $(\mathrm{A} 15)$ becomes

$$
P=2 \pi\left(\int_{0}^{a} E_{r 2} H_{\theta 2} r d r+\int_{a}^{b} E_{r 1} H_{\theta 1} r d r\right),
$$


where we have used the fact that $E_{r}$ and $H_{\theta}$ are azimuthally symmetric. Recalling that $E_{r}$ and $H_{\theta}$ can be written in the form

$$
E_{r}=\frac{1}{2} G e^{i(k z-\omega t)} e_{r}+\text { c.c. }, \quad H_{\theta}=\frac{1}{2} G e^{i(k z-\omega t)} h_{\theta}+\text { c.c. },
$$

the above can then be rewritten as

$$
\begin{aligned}
P= & \frac{\pi}{2}|G|^{2}\left(\int_{0}^{a}\left(e_{r_{2}} h_{\theta_{2}}^{*}+e_{r_{2}}^{*} h_{\theta_{2}}\right) r d r\right. \\
& \left.+\int_{a}^{b}\left(e_{r_{1}} h_{\theta_{1}}^{*}+e_{r_{1}}^{*} h_{\theta_{1}}\right) r d r\right) .
\end{aligned}
$$

Now, from (A8) and (A9)

$$
e_{r_{1,2}}=\frac{k}{\omega \epsilon_{0} \epsilon_{1,2}} h_{\theta_{1,2}}
$$

so

$$
P=\pi|G|^{2} \frac{k}{\omega \epsilon_{0}}\left(\int_{0}^{a} \frac{\left|h_{\theta 2}\right|^{2}}{\epsilon_{2}} r d r+\int_{a}^{b} \frac{\left|h_{\theta 1}\right|^{2}}{\epsilon_{1}} r d r\right) .
$$

Substituting for $h_{\theta_{1,2}}$ using (A8) and (A9) and setting $\epsilon_{2}$ $=1, \epsilon_{1}=\epsilon$ gives

$$
P=\frac{1}{2} \omega k \epsilon_{0} Q|G|^{2},
$$

where

$$
\begin{aligned}
Q= & \frac{\pi a^{2}}{\alpha_{2}^{2}}\left(I_{0}\left(\alpha_{2} a\right)^{2}-I_{1}\left(\alpha_{2} a\right)^{2}\right)-\frac{\pi \epsilon}{\kappa_{1}^{2}} \frac{J_{0}^{2}\left(\kappa_{2} a\right)}{\left(J_{0}\left(\kappa_{1} a\right) Y_{0}\left(\kappa_{1} b\right)-J_{0}\left(\kappa_{1} b\right) Y_{0}\left(\kappa_{1} a\right)\right)^{2}} \\
& \times\left[\left(J_{0}\left(\kappa_{1} a\right) Y_{0}\left(\kappa_{1} b\right)-J_{0}\left(\kappa_{1} b\right) Y_{0}\left(\kappa_{1} a\right)\right)^{2}+\left(J_{0}\left(\kappa_{1} b\right) Y_{1}\left(\kappa_{1} a\right)-J_{1}\left(\kappa_{1} a\right) Y_{0}\left(\kappa_{1} b\right)\right)^{2}\right] a^{2} \\
& +2\left[\left(J_{0}\left(\kappa_{1} b\right) Y_{1}\left(\kappa_{1} a\right)-J_{1}\left(\kappa_{1} a\right) Y_{0}\left(\kappa_{1} b\right)\right)\left(J_{0}\left(\kappa_{1} a\right) Y_{0}\left(\kappa_{1} b\right)-J_{0}\left(\kappa_{1} b\right) Y_{0}\left(\kappa_{1} a\right)\right)\right] \frac{a}{\kappa_{1}} \\
& \left.-\left[J_{0}\left(\kappa_{1} b\right) Y_{1}\left(\kappa_{1} b\right)-J_{1}\left(\kappa_{1} b\right) Y_{0}\left(\kappa_{1} b\right)\right]^{2} b^{2}\right]
\end{aligned}
$$

is a mode-dependent constant.

${ }^{1}$ M. A. Gundersen and G. Schaefer, Physics and Applications of Pseudosparks, NATO ASI Series B, 219 (Plenum, New York, 1990).

${ }^{2}$ K. Frank and J. Christiansen, IEEE Trans. Plasma Sci. 17, 748 (1989).

${ }^{3}$ G. F. Kirkman and M. A. Gundersen, Appl. Phys. Lett. 49, 494 (1986).

${ }^{4}$ H. Riege and E. Boggasch, IEEE Trans. Plasma Sci. 17, 775 (1989).

${ }^{5}$ E. Boggasch and M. J. Rhee, Appl. Phys. Lett. 56, 1746 (1990).

${ }^{6}$ W. Benker, J. Christiansen, K. Frank, H. Gundel, W. Hartmann, T. Redel, and M. Stetter, IEEE Trans. Plasma Sci. 17, 754 (1989).

${ }^{7}$ T. C. Marshall, Free Electron Lasers (Macmillan, New York, 1985).

${ }^{8}$ S. J. Cooke, A. W. Cross, W. He, and A. D. R. Phelps, Phys. Rev. Lett. 77, 4836 (1996).

${ }^{9}$ D. S. Lemons and L. E. Thode, Phys. Rev. Lett. 56, 2684 (1986).

${ }^{10}$ J. E. Walsh, T. C. Marshall, and S. Schleshinger, Phys. Fluids 20, 709 (1977).

${ }^{11}$ W. Peter and E. Garate, Phys. Rev. A 45, 8833 (1992).

${ }^{12}$ W. B. Case, R. D. Kaplan, J. E. Golub, and J. E. Walsh, J. Appl. Phys. 55, 2651 (1984).
${ }^{13}$ E. Garate, R. Cook, P. Heim, R. Layman, and J. E. Walsh, J. Appl. Phys. 58, 627 (1985).

${ }^{14}$ R. Liou, H. Figueroa, A. H. McCurdy, G. Kirkman-Amemiya, R. J. Temkin, H. Fetterman, and M. A. Gundersen, Appl. Phys. Lett. 61, 2779 (1992).

${ }^{15}$ K. Ramaswamy, W. W. Destler, and J. Rodgers, J. Appl. Phys. 83, 3514 (1998).

${ }^{16}$ H. Yin, W. He, G. R. M. Robb, A. D. R. Phelps, K. Ronald, and A. W. Cross, Phys. Rev. ST Accel. Beams 2, 020701 (1999).

${ }^{17}$ G. Schaefer and K. H. Schoenbach, in Ref. 1, Chap. 21, p. 55.

${ }^{18}$ P. Choi, R. Aliaga, and B. Blottiere, Appl. Phys. Lett. 63, 2750 (1993).

${ }^{19}$ M. P. Alberta, J. Derouard, L. C. Pitchford, N. Ouadoudi, and J. P. Boeuf, Phys. Rev. E 50, 2239 (1994).

${ }^{20}$ A. Anders, S. Anders, and M. A. Gundersen, Phys. Rev. Lett. 71, 364 (1993).

${ }^{21}$ H. P. Freund, Phys. Rev. Lett. 65, 2989 (1990). 